\title{
METALS REMOVED BY OUTFLOWS FROM MILKY WAY DWARF SPHEROIDAL GALAXIES*
}

\author{
Evan N. Kirby ${ }^{1,3}$, Crystal L. Martin $^{2}$, and Kristian Finlator ${ }^{2,3}$ \\ ${ }^{1}$ Department of Astronomy, California Institute of Technology, MC 249-17, Pasadena, CA 91125, USA \\ 2 Department of Physics, University of California, Santa Barbara, CA 93106, USA \\ Received 2011 September 26; accepted 2011 October 25; published 2011 November 8
}

\begin{abstract}
The stars in the dwarf spheroidal satellite galaxies (dSphs) of the Milky Way are significantly more metal-poor than would be expected from a closed box model of chemical evolution. Gas outflows likely carried away most of the metals produced by the dSphs. Based on previous Keck/DEIMOS observations and models, we calculate the mass in $\mathrm{Mg}, \mathrm{Si}, \mathrm{Ca}$, and $\mathrm{Fe}$ expelled from each of eight dSphs. Essentially, these masses are the differences between the observed amount of metals present in the dSphs' stars today and the inferred amount of metals produced by supernovae. We conclude that the dSphs lost $96 \%$ to $>99 \%$ of the metals their stars manufactured. We apply the observed mass function of Milky Way dSphs to the ejected mass function to determine that a single large dSph, like Fornax, lost more metals over $10 \mathrm{Gyr}$ than all smaller dSphs combined. Therefore, small galaxies like dSphs are not significant contributors to the metal content of the intergalactic medium. Finally, we compare our ejected mass function to previous X-ray measurements of the metal content of the winds from the post-starburst dwarf irregular galaxy NGC 1569. Remarkably, the most recent starburst in that galaxy falls exactly on the ejected-mass-stellarmass relation defined by the Milky Way dSphs.
\end{abstract}

Key words: galaxies: abundances - galaxies: dwarf - galaxies: evolution - intergalactic medium - Local Group Online-only material: color figures

\section{INTRODUCTION}

In a closed box model of chemical evolution (van den Bergh 1962; Schmidt 1963), the peak of the stellar metallicity distribution lies at the nucleosynthetic yield. For most supernovae ( $\mathrm{SNe})$, the nucleosynthetic yield is about solar metallicity or higher (Woosley \& Weaver 1995; Limongi \& Chieffi 2003; Nomoto et al. 2006). However, the peaks of the stellar metallicity distributions of all known dwarf spheroidal galaxies (dSphs) are less than solar (e.g., Aaronson \& Mould 1985; Buonanno et al. 1985). The dSphs likely lost metals during star formation, which reduced their effective nucleosynthetic yields (Hartwick 1976; Yoshii \& Arimoto 1987). Almost no dSph within $250 \mathrm{kpc}$ of the Milky Way (MW) contains any detectable gas at present (e.g., Blitz \& Robishaw 2000; Grcevich \& Putman 2009). Therefore, the missing metals are not hiding in the galaxies' interstellar medium. The metals have been lost.

SN winds drive gas out of galaxies, particularly small ones (Larson 1974; Dekel \& Silk 1986). It is possible to detect this gas flowing out of galaxies, but it is very difficult to measure the metallicity or even the mass of galactic winds. Absorption line studies of distant galaxies, including galaxies at cosmological redshift, can measure column densities and outflow velocities (Martin 2005; Rupke et al. 2005; Weiner et al. 2009; Steidel et al. 2010; Rubin et al. 2010) but not mass or composition. Smaller galaxies may be even more prone to gas outflows due to their shallow gravitational potential wells. Martin (1999) showed that the observed hot gas around gas-rich dwarf irregular galaxies (dIrrs) in the nearby universe must be escaping from those galaxies in the form of outflows. Just a few such galaxies are

\footnotetext{
* The data presented herein were obtained at the W.M. Keck Observatory, which is operated as a scientific partnership among the California Institute of Technology, the University of California, and the National Aeronautics and Space Administration. The Observatory was made possible by the generous financial support of the W. M. Keck Foundation.

3 Hubble Fellow.
}

suitable for X-ray spectroscopy to measure the composition of the escaping gas (e.g., NGC 1569; Martin et al. 2002).

Dwarf galaxies, which are numerous and have shallow gravitational potential wells, may be important contributors to the metal content of the intergalactic medium (IGM; Oppenheimer \& Davé 2008; Martin et al. 2010) or to their host galaxies. However, most nearby dwarf galaxies, especially dSphs, have not experienced appreciable star formation for several Gyr. As a result, it is even more difficult to observe gas expelled from Local Group dwarfs because it must have happened so long ago.

Kirby et al. (2010, 2011b) recently measured the stellar metal content of dSphs. The amount of metals produced over cosmic time for an individual dSph may be estimated from its stellar mass, an assumed stellar initial mass function (IMF), and theoretical SN yields and explosion rates. The difference between the inferred metal production by $\mathrm{SNe}$ and the current stellar metal content gives the amount of metals the galaxy has lost. This Letter presents that calculation and discusses the role of dSphs in enriching the IGM and their host galaxies.

\section{CALCULATIONS OF METALS EXPELLED}

Kirby et al. (2011a, hereafter K11a) constructed a basic chemical evolution model in order to explain the metallicity and $[\alpha / \mathrm{Fe}]$ distributions of eight MW dSphs: Fornax, Leo I, Leo II, Sculptor, Sextans, Draco, Canes Venatici I, and Ursa Minor. The model assumes that each galaxy begins its life with a certain amount of gas $\left(M_{g}(0)\right)$ but no stars. Primordial, metal-free gas flows into the galaxy at a prescribed inflow rate $\left(d M_{g} / d t=A_{\text {in }} t e^{-t / \tau_{\text {in }}}\right)$. Stars form according to a star formation rate law $\left(d M_{*} / d t=A_{*} M_{g}^{\alpha}\right)$. Type II SNe explode from massive $\left(10-100 M_{\odot}\right)$ stars and Type Ia $\mathrm{SNe}$ explode according to an empirical delay time distribution (Maoz et al. 2010). Most important, for this Letter, the galaxy loses a fixed amount of gas $\left(A_{\text {out }}\right)$ during each $\mathrm{SN}$ explosion. K11a varied the parameters $M_{g}(0), A_{\text {in }}, A_{*}, A_{\text {out }}, \tau_{\text {in }}$, and $\alpha$ until 
Table 1

Inferred Ejected Masses Over $10 \mathrm{Gyr}$

\begin{tabular}{|c|c|c|c|c|c|c|}
\hline $\mathrm{dSph}$ & $\begin{array}{c}L \\
\left(L_{\odot}\right)\end{array}$ & $\begin{array}{c}M_{*} \\
\left(M_{\odot}\right)\end{array}$ & $\begin{array}{c}M_{\mathrm{Mg}} \\
\left(M_{\odot}\right)\end{array}$ & $\begin{array}{c}M_{\mathrm{Si}} \\
\left(M_{\odot}\right)\end{array}$ & $\begin{array}{c}M_{\mathrm{Ca}} \\
\left(M_{\odot}\right)\end{array}$ & $\begin{array}{c}M_{\mathrm{Fe}} \\
\left(M_{\odot}\right)\end{array}$ \\
\hline Fornax & $(1.8 \pm 0.5) \times 10^{7}$ & $(1.9 \pm 0.5) \times 10^{7}$ & $1.2 \times 10^{4}$ & $2.9 \times 10^{4}$ & $1.9 \times 10^{3}$ & $8.3 \times 10^{4}$ \\
\hline Leo I & $(5.6 \pm 1.7) \times 10^{6}$ & $(4.6 \pm 1.4) \times 10^{6}$ & $3.7 \times 10^{3}$ & $7.9 \times 10^{3}$ & $4.9 \times 10^{2}$ & $2.1 \times 10^{4}$ \\
\hline Sculptor & $(2.3 \pm 1.1) \times 10^{6}$ & $(1.2 \pm 0.6) \times 10^{6}$ & $9.9 \times 10^{2}$ & $2.1 \times 10^{3}$ & $1.3 \times 10^{2}$ & $5.5 \times 10^{3}$ \\
\hline Leo II & $(6.6 \pm 1.9) \times 10^{5}$ & $(1.4 \pm 0.4) \times 10^{6}$ & $1.2 \times 10^{3}$ & $2.7 \times 10^{3}$ & $1.7 \times 10^{2}$ & $7.1 \times 10^{3}$ \\
\hline Sextans & $(4.1 \pm 1.2) \times 10^{5}$ & $(8.5 \pm 2.4) \times 10^{5}$ & $6.6 \times 10^{2}$ & $1.5 \times 10^{3}$ & $9.2 \times 10^{1}$ & $3.8 \times 10^{3}$ \\
\hline Draco & $(2.7 \pm 0.4) \times 10^{5}$ & $(9.1 \pm 1.4) \times 10^{5}$ & $7.0 \times 10^{2}$ & $1.6 \times 10^{3}$ & $9.7 \times 10^{1}$ & $4.0 \times 10^{3}$ \\
\hline Can. Ven. I & $(2.3 \pm 0.4) \times 10^{5}$ & $(6.3 \pm 1.1) \times 10^{5}$ & $4.9 \times 10^{2}$ & $1.1 \times 10^{3}$ & $6.8 \times 10^{1}$ & $2.8 \times 10^{3}$ \\
\hline Ursa Minor & $(2.2 \pm 0.7) \times 10^{5}$ & $(5.6 \pm 1.7) \times 10^{5}$ & $3.9 \times 10^{2}$ & $9.1 \times 10^{2}$ & $5.5 \times 10^{1}$ & $2.3 \times 10^{3}$ \\
\hline
\end{tabular}

References. $L$ (luminosity): Martin et al. (2008) for Canes Venatici I; Irwin \& Hatzidimitriou (1995) otherwise. $M_{*}$ (stellar mass): Woo et al. (2008), except that we assumed that Canes Venatici I the same $M_{*} / L$ as Ursa Minor.

the modeled metallicity and $[\alpha / \mathrm{Fe}]$ distributions matched the observed distributions.

This model provides good matches to the abundance distributions of the less luminous dSphs. The inferred star formation histories also were qualitatively consistent with histories measured from color-magnitude diagrams. Essentially, nearly all stars in the less luminous dSphs are ancient and they formed in 2 Gyr or less. However, the models for the most luminous dSphs (Fornax, Leo I, and to some extent, Leo II) have chemically measured star formation durations of only $\sim 1$ Gyr. The color-magnitude diagrams for these galaxies rule out such short star formation lifetimes and also rule out single bursts of star formation. These dSphs experienced several epochs of an increase in star formation rate (SFR) followed by a decrease. The K11a models permit only one episode of star formation (a single rise and fall of SFR). Therefore, the models are imperfect descriptions of Fornax, Leo I, and possibly Leo II.

On the other hand, for an old enough population, such as the MW dSphs, most of the SNe that will ever explode have already exploded. Therefore, the star formation history (SFR as a function of time) has a small effect on the total metal production integrated over cosmic time. Because SN yields are metallicity dependent, the star formation history does matter to a small degree.

As a supplement to the work of K11a, we compute the mass in a number of metals, $M_{\text {ejected }}$, that the dSphs lost to SN winds. The K11a chemical evolution model tracks the amount and composition of expelled gas with each 1 Myr time step. The model was terminated when the dSphs were exhausted of gas. Although dSphs lose a small fraction of stars to tidal interactions with the MW (Majewski et al. 2000), we neglect this deduction from the final stellar mass. The existence of the luminosity-metallicity relation (Mateo 1998; Kirby et al. 2011b) limits the severity of tidal stripping of luminous matter (also see Peñarrubia et al. 2008) because tidally stripped versions of massive satellites would be just as metal-rich as other massive satellites, but small, metal-rich satellites do not exist.

In order to mitigate the uncertainties imposed on $M_{\text {ejected }}$ from uncertain star formation histories, we advance the chemical evolution model for each $\mathrm{dSph}$ to an age of $10 \mathrm{Gyr}$. We compute the amount of ejecta-mostly in Fe-from Type Ia SNe over $10 \mathrm{Gyr}$, even though star formation ends in the models long before then. Like K11a, we use Nomoto et al.'s (2006) nucleosynthetic yields for Type II SNe, Iwamoto et al.'s (1999) yields for Type Ia SNe, and Maoz et al.'s (2010) delay time distribution for Type Ia SNe. The first Type Ia SN explodes 100 Myr after the birth of its progenitor, and the Type Ia SN rate declines as $t^{-1.1}$. Based on the theoretical yields and IMF (Kroupa et al. 1993) we have adopted, Type Ia SNe produce 94\% of the iron over 10 Gyr. Asymptotic giant branch stars are included in the chemical evolution model, but at these low metallicities, they are responsible for only a few percent of the total production of $\mathrm{Mg}, \mathrm{Si}, \mathrm{Ca}$, and $\mathrm{Fe}$ (Karakas 2010).

Table 1 gives $M_{\text {ejected }}$ for $\mathrm{Mg}, \mathrm{Si}, \mathrm{Ca}$, and $\mathrm{Fe}$ for the eight MW dSphs in our sample. We do not attempt to estimate errors on $M_{\text {ejected }}$ because the dominant source of uncertainty is the SN yields. Differences between different yield models (e.g., Woosley \& Weaver 1995; Nomoto et al. 2006) can be a factor of several for some elements.

If Type Ia SNe have a prompt component (Scannapieco \& Bildsten 2005), then the Type Ia SNe at early times could feed the same wind as the Type II SNe. However, the delayed Type Ia $\mathrm{SNe}$ at late times explode at a low rate. Without continuous explosions, it may not be possible to drive a wind. Nonetheless, nearly all dSphs are free of gas. Therefore, the "missing metals"- the difference between the current metal content of the dSph and the amount of metals estimated to have been produced by all of the dSph's $\mathrm{SNe}$ - have left the dSphs, whether in coherent winds or otherwise.

K11a assumed that the composition of the gas lost to SN winds was the same as the gas within the galaxy at the time of loss. However, outflows could be metal-enhanced, especially because the SNe that expel gas from the galaxy are the same events that enrich the galaxy with metals (Vader 1986; Mac Low \& Ferrara 1999). The mass of the outflow is related inversely to its unknown metallicity, and K11a showed that increasing the outflowing metallicity decreased the total amount of galactic mass loss required by as much as a factor of 40 . Given this uncertainty, we estimate only the mass in ejected metals and not the total mass of ejected material. The uncertainty in the metallicity of the outflows does not extend to the independent estimate of the ejected metal mass.

\section{RELATIONS WITH STELLAR MASS}

\subsection{Total Metal Output}

Figure 1 shows the mass in each of several metals lost to SN winds summed over 10 Gyr. Not surprisingly, the dSphs with larger stellar masses eject more metals than the dSphs with smaller stellar masses. The $M_{\text {ejected }}-M_{*}$ relation is linear, which is the result expected if the dSphs lost all of their metals. The intercepts of the best-fit lines indicate that dSphs lost a constant $0.5 \%$ of their final stellar masses in the form of $\mathrm{Fe}, 0.2 \%$ as $\mathrm{Si}$, $0.1 \%$ as $\mathrm{Mg}$, and $0.01 \%$ as $\mathrm{Ca}$. 


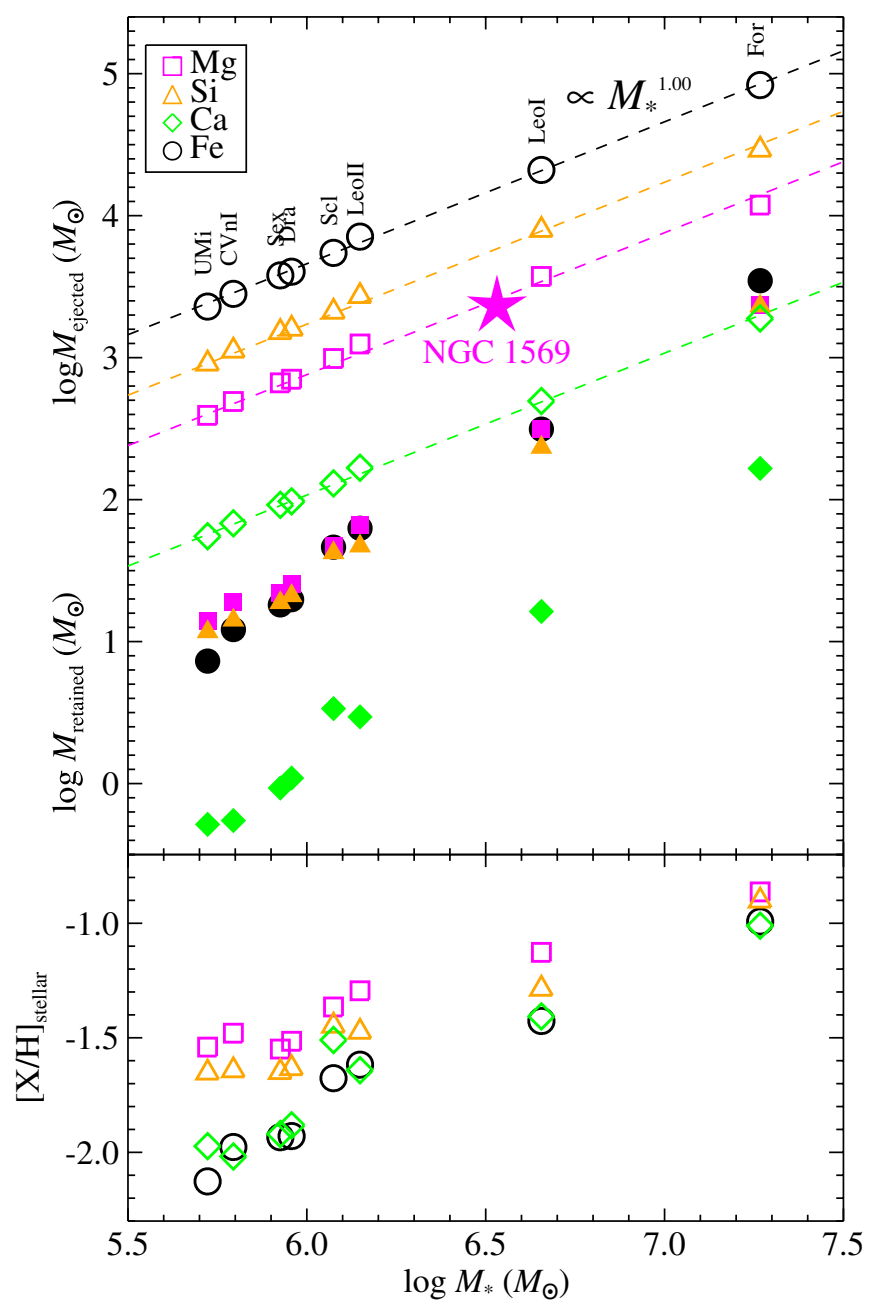

Figure 1. Top: the mass in four different metals inferred to have been lost from (hollow points) or retained in (filled points) MW dSphs as a function of their present stellar mass. The hollow points show the "missing metals," which should have been produced by the SNe in these dSphs but are not present in the stars. The masses of ejected metals include the ejecta of Type Ia SNe that exploded up to $10 \mathrm{Gyr}$ after the cessation of star formation. The dashed lines illustrate slopes of $M_{*}^{1.00}$, which is the expected relation if dSphs lost all of the metals they produced. The large star shows the Mg mass in the current outflow from NGC 1569 vs. the stellar mass of the most recent starburst (Martin et al. 2002). Bottom: the stellar mass-metallicity relation for the stars in dSphs (Kirby et al. $2011 b)$. Metallicity is given separately as $[\mathrm{Fe} / \mathrm{H}],[\mathrm{Mg} / \mathrm{H}],[\mathrm{Si} / \mathrm{H}]$, and $[\mathrm{Ca} / \mathrm{H}]$.

(A color version of this figure is available in the online journal.)

Of course, the dSphs could not have truly lost all of their metals because the stars are not metal free. However, the amount of metals retained $\left(M_{\text {retained }}\right.$, the filled points in Figure 1) in stars is tiny compared to the amount of metals produced. Kirby et al. (2011b) measured the average metallicity of Fornax to be $\langle[\mathrm{Fe} / \mathrm{H}]\rangle=-1.0 .^{4}$ Given its stellar mass of $1.9 \times$ $10^{7} M_{\odot}$, Fornax's stars contain $4 \times 10^{3} M_{\odot}$ of Fe. Even though Fornax retained the highest fraction of Fe of all eight dSphs considered here, the galaxy presently has only $4 \%$ of the Fe that it produced. In Ursa Minor, this fraction is only $0.2 \%$.

The bottom panel of Figure 1 expresses metal retention in another way. The black circles show the stellar-mass-ironmetallicity relation for MW dSphs (Kirby et al. 2011b). The

\footnotetext{
4 We adopt the solar abundance of $\mathrm{Fe}$ as $12+\log (n(\mathrm{Fe}) / n(\mathrm{H}))=7.52$ (Sneden et al. 1992). We adopt 7.58, 7.55, and 6.36 for $\mathrm{Mg}$, Si, and $\mathrm{Ca}$, respectively (Anders \& Grevesse 1989).
}

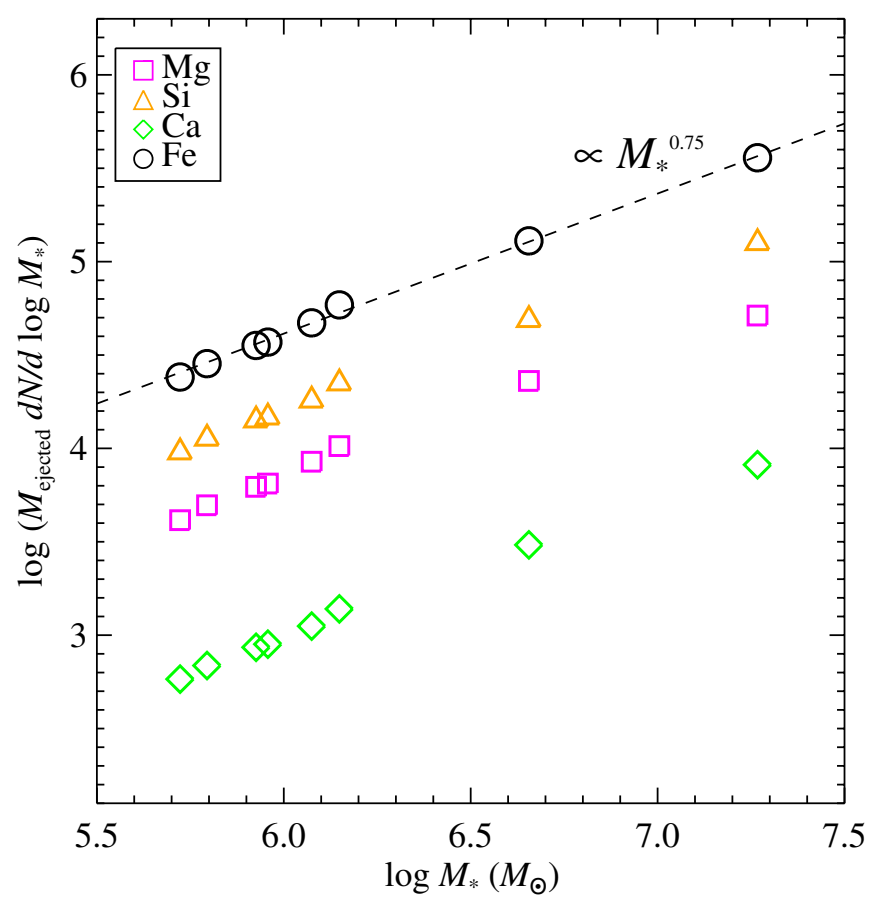

Figure 2. Metals ejected from dSphs convolved with the luminosity function for MW dSphs $\left(d N / d \log M_{*} \propto M_{*}^{-0.25}\right.$; Koposov et al. 2008). The dashed line shows $M_{*}^{0.75}$. The axes are logarithmic, and the metal output from small dSphs is negligible compared to large dSphs.

(A color version of this figure is available in the online journal.)

other points show the mass-metallicity relations for other elements. The smaller dSphs retain a larger fraction of $\alpha$ elements relative to iron than the larger dSphs. The dependence on stellar mass indicates that the larger dSphs formed stars for longer and therefore experienced a higher ratio of Type Ia to Type II SNe.

\subsection{Relative Metal Output}

The mass-metallicity relation indicates that the more massive $\mathrm{dSphs}$ retained more of their metals in stars rather than losing them to galactic winds. Therefore, the less massive dSphs contribute a larger fraction of their metal mass to the IGM or to their host galaxies. To further explore the importance of small dSphs, we convolve the ejected mass function with the stellar mass function for MW dSphs. Koposov et al. (2008) calculated the luminosity function of MW dSphs by correcting the observed Sloan Digital Sky Survey luminosity function for sky coverage and bias against low surface brightness dSphs. The result was $d N / d M_{V}=10 \times 10^{0.1\left(M_{V}+5\right)}$. Assuming the stellar-mass-to-light ratio for an old population, $M_{*} / L=2$, the relation becomes $d N / d \log M_{*}=284 M_{*}^{-0.25}$, where $M_{*}$ is measured in $M_{\odot}$.

Figure 2 shows the product of this dSph mass function and the ejected mass function from Figure 1. Even though the small $\mathrm{dSphs}$ are more numerous, they are not significant contributors to IGM metals. Even if the relation for the smaller dSphs is extrapolated to zero mass, then all dSphs the size of Leo I or smaller contributed just $7.5 \times 10^{4} M_{\odot}$ of Fe to the IGM over 10 Gyr. Fornax alone provided more Fe, and even more massive galaxies likely make Fornax's contribution negligible. The same argument applies to the other elements as well. 


\section{3. $[\alpha / F e]$}

Averaged over a long enough time, the ratio of the numbers of Type II to Type Ia SNe asymptotes to the same value in all dSphs as long as the IMF is the same. As a result, the $[\alpha / \mathrm{Fe}]$ ratios of ejecta from $\mathrm{dSphs}$ integrated over $10 \mathrm{Gyr}$ are not functions of stellar mass. Expressed relative to the solar ratios, the $10 \mathrm{Gyr}$ ratios for our adopted $\mathrm{SN}$ yields and our adopted IMF are $[\mathrm{Mg} / \mathrm{Fe}]=-0.45,[\mathrm{Si} / \mathrm{Fe}]=-0.15$, and $[\mathrm{Ca} / \mathrm{Fe}]=-0.30$.

\section{COMPARISON TO NGC 1569}

Mayer et al. (2006) theorized that gas-rich dIrrs can be converted into gas-free dSphs by interaction with their host galaxies. Tidal stirring can turn disks into spheroids, and ram pressure stripping - in addition to SN-driven outflow-can remove all of the gas. With this relationship between dIrrs and dSphs in mind, we compare our inferences for the metals ejected from dSphs to the observed metal content of the wind presently flowing out the post-starburst dIrr NGC 1569 $\left(M_{*}=1.8 \times 10^{8} M_{\odot} ;\right.$ Israel 1988).

Martin et al. (2002) estimated that the most recent starburst in that galaxy ejected $M_{\mathrm{O}} \approx 3.4 \times 10^{4} M_{\odot}$ of oxygen. They based this estimate on an X-ray spectral fit to several $\alpha$ elements, including $\mathrm{O}$ and $\mathrm{Mg}$. In order to compare to our own ejected metal estimates, we note that $M_{\mathrm{O}}$ is related to $M_{\mathrm{Mg}}$ by a multiplicative factor (about 15, which is also the solar ratio) because both elements are formed in similar nucleosynthetic processes in massive stars. Therefore, the starburst must have ejected $M_{\mathrm{Mg}} \approx 2.3 \times 10^{3} M_{\odot}$ of magnesium. The mass of stars formed in the burst was $M_{*} \approx 3.4 \times 10^{6} M_{\odot}$ over $10-20$ Myr.

NGC 1569 is more massive than any of the dSphs we consider, and the SFR in the burst was much higher than the average rates in the dSphs. Nonetheless, the outflowing magnesium mass from the recent burst in NGC 1569 agrees remarkably well with the relation between $M_{\mathrm{Mg}}$ and $M_{*}$ defined by MW dSphs. Figure 1 shows that the NGC 1569 outflow falls exactly in line between Leo I and Leo II. Of course, we must keep in mind that we are considering only the very recent starburst and not the entire galaxy, which is 50 times more massive. However, only the ejecta of the current starburst is directly measurable, and the measurement is uncertain by a factor of at least two.

Martin et al. (2002) also measured the $[\alpha / \mathrm{Fe}]$ ratio to be 0.3-0.6 (2-4 times solar). This value is larger than what we infer for the MW dSphs. However, the winds flowing out of NGC 1569 came from a very short burst-likely short enough that Type Ia SN ejecta is not present in the winds. Therefore, the $[\alpha / \mathrm{Fe}]$ ratios merely reflect the fact that the star formation was intense in the recent burst in NGC 1569 but not in the dSphs.

Martin et al.'s completely independent method for measuring the metal content of galactic winds has placed a different, though possibly related, type of dwarf galaxy on the same $M_{\text {ejected }}-M_{*}$ relation as the one defined by MW dSphs. This result has several implications. First, NGC 1569 retained almost none of the metals produced in its recent starburst, a result that Martin et al. found from their own chemical evolution model. Second, the theoretical SN yields are accurate enough to place the observed magnesium mass ejected from NGC 1569 on the same relation as the masses ejected from dSphs, which were calculated from the theoretical yields. Finally, even a galaxy as massive as NGC $1569\left(\sigma_{v}=21 \mathrm{~km} \mathrm{~s}^{-1}\right.$, Stil \& Israel 2002, compared to $10 \mathrm{~km} \mathrm{~s}^{-1}$ for a typical dSph) loses nearly all of the metals it produces.

\section{DISCUSSION}

The possibility that the majority of metals that currently reside in the IGM were originally ejected by low-mass systems (Oppenheimer \& Davé 2008; Martin et al. 2010) emphasizes the need for constraints on models for galactic outflows. Galaxy evolution models (e.g., Somerville et al. 2001; Oppenheimer \& Davé 2006) generally assume that star-forming galaxies drive outflows whose metallicity tracks the interstellar medium and whose outflowing mass is proportional to $M_{*} / \sigma^{\alpha}$. Here, $\sigma$ is the velocity dispersion that scales with the halo mass $\left(\sigma \propto M_{h}^{1 / 3}\right)$ and $\alpha$ is a scaling parameter. Setting $\alpha=1$ yields a momentumdriven scaling and $\alpha=2$ yields an energy-driven scaling. Some models (e.g., Bower et al. 2006) invoke a stronger scaling $(\alpha=3)$ in order to match the faint end of the luminosity function. Let us assume that the metallicity of outflowing gas $Z_{W}$ tracks the stellar metallicity $Z_{*}$, which in turn scales with stellar mass as $M_{*}^{0.31}$ (Kirby et al. 2011b). Let us further assume the stellar-mass-halo-mass scaling that is observed at low masses $\left(M_{*} \propto M_{h}^{2.17}\right.$; Leauthaud et al. 2011). The mass in ejected metals is the outflow mass multiplied by the wind metallicity. Therefore, the model assumptions correspond to the assumption that $M_{\text {ejected }} \propto\left(M_{*} / \sigma^{\alpha}\right) Z_{W}=M_{*}^{1.31} / M_{h}^{\alpha / 3}=M_{*}^{1.31-\alpha / 6.51}$, or $M_{*}^{1.16}$ and $M_{*}^{1.00}$ for momentum-driven and energy-driven scalings, respectively. Taken at face value, our observation of the ejected mass relation, $M_{\text {ejected }} \propto M_{*}^{1.00}$, favors an energydriven scaling.

We emphasize that these inferences depend strongly on the assumed stellar-mass-halo-mass relation as well as the metallicities within the outflows. They also neglect many physical processes, such as tidal stripping, that could preferentially affect low-mass systems. Furthermore, much of the metals from Type Ia SNe are lost well after the cessation of star formation and well after the galaxies are devoid of interstellar gas. That makes the distinction between energy- and momentum-driven winds poorly defined. These uncertainties emphasize the need for improved observational constraints on the kinematics of low-mass systems as well as the mass-loading and metal mass fractions of outflows from low-mass galaxies.

The biggest limitation of our approach at measuring $M_{\text {ejected }}$ is that we have measured the mass only in metals. We provide no constraints on the total outflowing mass, including hydrogen. Therefore, we also provide no constraints on the metal fraction of the outflows. One way to constrain the metallicity is to fix the total mass participating in gas flows and star formation. For instance, we could assume that the baryonic mass associated with a dSph obeys the cosmic-baryon-to-dark-matter ratio. Unfortunately, the disconnect between stellar and dark matter masses (Mateo 1998; Strigari et al. 2008) and the tidal interactions (Peñarrubia et al. 2008) of MW dSphs make it impossible to estimate their total, original dark matter masses.

Semi-analytic models (SAMs; e.g., Cooper et al. 2010) may provide a path forward. By treating a variety of processes that likely affected dSph growth, such as tidal stripping, pre-enriched inflows, and returning outflows, SAMs provide a flexible but complete account of how outflows impact metallicities. A model galaxy whose chemical abundances match observed abundance distributions (Kirby et al. 2011a, 2011b) and our inferred ejected metal masses might inform us about these processes. We encourage interested modelers to interrogate their existing SAMs for unique constraints that our observations place on the way in which low-mass galaxies process their gas into outflows, stars, and evolving gas reservoirs. 
We thank J. Cohen and the anonymous referee for careful, constructive criticism that improved this Letter. Support for this work was provided by NASA through Hubble Fellowship grants 51256.01 awarded to E.N.K. and 51254.01 awarded to K.F. by the Space Telescope Science Institute, which is operated by the Association of Universities for Research in Astronomy, Inc., for NASA, under contract NAS 5-26555. Support to C.L.M. was provided through NSF Grant AST-080816.

Facility: Keck:II (DEIMOS)

\section{REFERENCES}

Aaronson, M., \& Mould, J. 1985, ApJ, 290, 191

Anders, E., \& Grevesse, N. 1989, Geochim. Cosmochim. Acta, 53, 197 Blitz, L., \& Robishaw, T. 2000, ApJ, 541, 675

Bower, R. G., Benson, A. J., Malbon, R., et al. 2006, MNRAS, 370, 645

Buonanno, R., Corsi, C. E., Fusi Pecci, F., Hardy, E., \& Zinn, R. 1985, A\&A, 152,65

Cooper, A. P., Cole, S., Frenk, C. S., et al. 2010, MNRAS, 406, 744

Dekel, A., \& Silk, J. 1986, ApJ, 303, 39

Grcevich, J., \& Putman, M. E. 2009, ApJ, 696, 385

Hartwick, F. D. A. 1976, ApJ, 209, 418

Irwin, M., \& Hatzidimitriou, D. 1995, MNRAS, 277, 1354

Israel, F. P. 1988, A\&A, 194, 24

Iwamoto, K., Brachwitz, F., Nomoto, K., et al. 1999, ApJS, 125, 439

Karakas, A. I. 2010, MNRAS, 403, 1413

Kirby, E. N., Cohen, J. G., Smith, G. H., et al. 2011a, ApJ, 727, 79 (K11a)

Kirby, E. N., Guhathakurta, P., Simon, J. D., et al. 2010, ApJS, 191, 352

Kirby, E. N., Lanfranchi, G. A., Simon, J. D., Cohen, J. G., \& Guhathakurta, P. 2011b, ApJ, 727, 78

Koposov, S., Belokurov, V., Evans, N. W., et al. 2008, ApJ, 686, 279

Kroupa, P., Tout, C. A., \& Gilmore, G. 1993, MNRAS, 262, 545
Larson, R. B. 1974, MNRAS, 169, 229

Leauthaud, A., Tinker, J., Bundy, K., et al. 2011, arXiv:1104.0928

Limongi, M., \& Chieffi, A. 2003, ApJ, 592, 404

Mac Low, M.-M., \& Ferrara, A. 1999, ApJ, 513, 142

Majewski, S. R., Ostheimer, J. C., Patterson, R. J., et al. 2000, AJ, 119, 760

Maoz, D., Sharon, K., \& Gal-Yam, A. 2010, ApJ, 722, 1879

Martin, C. L. 1999, ApJ, 513, 156

Martin, C. L. 2005, ApJ, 621, 227

Martin, C. L., Kobulnicky, H. A., \& Heckman, T. M. 2002, ApJ, 574, 663

Martin, C. L., Scannapieco, E., Ellison, S. L., et al. 2010, ApJ, 721, 174

Martin, N. F., de Jong, J. T. A., \& Rix, H.-W. 2008, ApJ, 684, 1075

Mateo, M. L. 1998, ARA\&A, 36, 435

Mayer, L., Mastropietro, C., Wadsley, J., Stadel, J., \& Moore, B. 2006, MNRAS, 369, 1021

Nomoto, K., Tominaga, N., Umeda, H., Kobayashi, C., \& Maeda, K. 2006, Nucl. Phys. A, 777, 424

Oppenheimer, B. D., \& Davé, R. 2006, MNRAS, 373, 1265

Oppenheimer, B. D., \& Davé, R. 2008, MNRAS, 387, 577

Peñarrubia, J., Navarro, J. F., \& McConnachie, A. W. 2008, ApJ, 673, 226

Rubin, K. H. R., Weiner, B. J., Koo, D. C., et al. 2010, ApJ, 719, 1503

Rupke, D. S., Veilleux, S., \& Sanders, D. B. 2005, ApJS, 160, 87

Scannapieco, E., \& Bildsten, L. 2005, ApJ, 629, L85

Schmidt, M. 1963, ApJ, 137, 758

Sneden, C., Kraft, R. P., Prosser, C. F., \& Langer, G. E. 1992, AJ, 104, 2121

Somerville, R. S., Primack, J. R., \& Faber, S. M. 2001, MNRAS, 320, 504

Steidel, C. C., Erb, D. K., Shapley, A. E., et al. 2010, ApJ, 717, 289

Stil, J. M., \& Israel, F. P. 2002, A\&A, 392, 473

Strigari, L. E., Bullock, J. S., Kaplinghat, M., et al. 2008, Nature, 454, 1096

Vader, J. P. 1986, ApJ, 305, 669

van den Bergh, S. 1962, AJ, 67, 486

Weiner, B. J., Coil, A. L., Prochaska, J. X., et al. 2009, ApJ, 692, 187

Woo, J., Courteau, S., \& Dekel, A. 2008, MNRAS, 390, 1453

Woosley, S. E., \& Weaver, T. A. 1995, ApJS, 101, 181

Yoshii, Y., \& Arimoto, N. 1987, A\&A, 188, 13 